\title{
Semi-orthogonal wavelet frames on positive half-line using the Walsh Fourier transform
}

\author{
Abdullah Abdullah and Afroz Afroz \\ Department of Mathematics, Zakir Husain Delhi College, (University of Delhi), New Delhi, India \\ Department of Mathematics, School of sciences, Maulana Azad National Urdu University, Hyderabad, India
}

Received: 11 February 2017, Accepted: 12 April 2017

Published online: 2 May 2018.

\begin{abstract}
We investigate semi-orthogonal wavelet frames on positive half-line and provide a characterization of frame wavelets by means of some basic equations in the frequency domain.
\end{abstract}

Keywords: Frame multiresolution analysis, wavelet frame, semi-orthogonality, Walsh Fourier transform.

\section{Introduction}

The concept of frames in a Hilbert space was originally introduced by Duffin and Schaeffer [7] in the context of non-harmonic Fourier series. In signal processing, this concept has become very useful in analyzing the completeness and stability of linear discrete signal representations. Frames did not seem to generate much interest until the ground-breaking work of Daubechies et al. [6]. They combined the theory of continuous wavelet transforms with the theory of frames to introduce wavelet (affine) frames for $L^{2}(\mathbb{R})$. Since then the theory of frames began to be more widely investigated, and now it is found to be useful in signal processing, image processing, harmonic analysis, sampling theory, data transmission with erasures, quantum computing and medicine. Recently, more applications of the theory of frames are found in diverse areas including optics, filter banks, signal detection and in the study of Bosev spaces and Banach spaces. We refer [5] for an introduction to frame theory and its applications.

In recent years, wavelets have been generalized in many different settings, for example locally compact abelian group, abstract Hilbert spaces, locally compact Cantor dyadic group, Vilenkin group, local fields and positive half-line. In this paper our interest is in positive half-line. Farkov [8] has given general construction of compactly supported orthogonal $p$-wavelets in $L^{2}\left(\mathbb{R}^{+}\right)$. An algorithm for biorthogonal wavelets related to Walsh functions on positive half line was given in [9]. Dyadic wavelet frames on the positive half-line R+ were constructed by Shah and Debnath in [15] using Walsh Fourier transforms. They have established necessary and sufficient conditions for the system $\left\{\psi_{j, k}(x)=2^{j / 2} \psi\left(2^{j} x \ominus k\right), j \in \mathbb{Z}, k \in \mathbb{Z}^{+}\right\}$to be a frame for $L^{2}\left(\mathbb{R}^{+}\right)$. Wavelet frame packets related to the Walsh polynomials were deeply investigated by Shah and Debnath in [16]. A constructive procedure for constructing tight wavelet frames on positive half-line using extension principles was recently considered by Shah in [17], in which he has pointed out a method for constructing affine frames in $L^{2}\left(\mathbb{R}^{+}\right)$. Moreover, the author has established sufficient conditions for a finite number of functions to form a tight affine frames for $L^{2}\left(\mathbb{R}^{+}\right)$. Abdullah [1] has given characterization of nonuniform wavelet sets on positive half-line and Characterization of wavelets on positive half line by means of two basic equations in the Fourier domain was established in [2].

*Corresponding author e-mail: abd.zhc.du@gmail.com; afrozjmi123@gmail.com

() 2018 BISKA Bilisim Technology 
In this paper, we extend the notion of wavelet frames to semi-orthogonal wavelet frames on positive half-line using the Walsh Fourier transform. This paper is organized as follows: In Sec. 2, we present a brief review of generalized Walsh functions and polynomials, the Walsh Fourier transform and FMRA in $L^{2}\left(\mathbb{R}^{+}\right)$. A characterization of semi-orthogonal wavelet frames on positive half-line is given in Section 3.

\section{Notations and preliminaries}

Let $p$ be a fixed natural number greater than 1 . As usual, let $\mathbb{R}^{+}=[0, \infty)$ and $\mathbb{Z}^{+}=\{0,1, \ldots\}$. Denote by $[x]$ the integer part of $x$. For $x \in \mathbb{R}^{+}$and for any positive integer $j$, we set

$$
x_{j}=\left[p^{j} x\right](\bmod p), x_{-j}=\left[p^{1-j} x\right](\bmod p),
$$

where $x_{j}, x_{-j} \in\{0,1, \ldots, p-1\}$.

Consider the addition defined on $\mathbb{R}^{+}$as follows

$$
x \oplus y=\sum_{j<0} \xi_{j} p^{-j-1}+\sum_{j>0} \xi_{j} p^{-j}
$$

with

$$
\xi_{j}=x_{j}+y_{j}(\bmod p), \quad j \in \mathbb{Z} \backslash\{0\}
$$

where $\xi_{j} \in\{0,1,2, \ldots, p-1\}$ and $x_{j}, y_{j}$ are calculated by (1). Moreover, we write $z=x \ominus y$ if $z \oplus y=x$.

For $x \in[0,1)$, let $r_{0}(x)$ be given by

$$
r_{0}(x)=\left\{\begin{array}{l}
1, x \in\left[0, \frac{1}{p}\right) \\
\varepsilon_{p}^{j}, x \in\left[j p^{-1},(j+1) p^{-1}\right), j=1,2, \ldots, p-1,
\end{array}\right.
$$

where $\varepsilon_{p}=\exp \left(\frac{2 \pi i}{p}\right)$. The extension of the function $r_{0}$ to $\mathbb{R}^{+}$is defined by the equality $r_{0}(x+1)=r_{0}(x), x \in \mathbb{R}^{+}$. Then the generalized Walsh functions $\left\{\omega_{m}(x)\right\}_{m \in \mathbb{Z}^{+}}$are defined by

$$
\omega_{0}(x)=1, \quad \omega_{m}(x)=\prod_{j=0}^{k}\left(r_{0}\left(p^{j} x\right)\right)^{\mu_{j}}
$$

where $m=\sum_{j=0}^{k} \mu_{j} p^{j}, \mu_{j} \in\{0,1,2, \ldots, p-1\}, \mu_{k} \neq 0$. For $x, \omega \in \mathbb{R}^{+}$, let

$$
\chi(x, \omega)=\exp \left(\frac{2 \pi i}{p} \sum_{j=1}^{\infty}\left(x_{j} \omega_{-j}+x_{-j} \omega_{j}\right)\right)
$$

where $x_{j}$ and $\omega_{j}$ are calculated by (1). We observe that

$$
\chi\left(x, \frac{m}{p^{n-1}}\right)=\chi\left(\frac{x}{p^{n-1}}, m\right)=\omega_{m}\left(\frac{x}{p^{n-1}}\right) \quad \forall x \in\left[0, p^{n-1}\right), m \in \mathbb{Z}^{+}
$$


The Walsh Fourier transform of a function $f \in L^{1}\left(\mathbb{R}^{+}\right)$is defined by

$$
\tilde{f}(\omega)=\int_{\mathbb{R}^{+}} f(x) \overline{\chi(x, \omega)} d x
$$

where $\chi(x, \omega)$ is given by (5). If $f \in L^{2}\left(\mathbb{R}^{+}\right)$and

$$
J_{a} f(\omega)=\int_{0}^{a} f(x) \overline{\chi(x, \omega)} d x \quad(a<0),
$$

then $\tilde{f}$ is defined as limit of $J_{a} f$ in $L^{2}\left(\mathbb{R}^{+}\right)$as $a \rightarrow \infty$.

The properties of Walsh Fourier transform are quite similar to the classical Fourier transform. It is known that systems $\{\chi(\alpha, .)\}_{\alpha=0}^{\infty}$ and $\{\chi(., \alpha)\}_{\alpha=0}^{\infty}$ are orthonormal bases in $L^{2}(0,1)$. Let us denote by $\{\omega\}$ the fractional part of $\omega$. For $l \in \mathbb{Z}^{+}$, we have $\chi(l, \omega)=\chi(l,\{\omega\})$.

If $x, y, \omega \in \mathbb{R}^{+}$and $x \oplus y$ is $p$-adic irrational, then

$$
\chi(x \oplus y, \omega)=\chi(x, \omega) \chi(y, \omega), \quad \chi(x \ominus y, \omega)=\chi(x, \omega) \overline{\chi(y, \omega)} .
$$

For given $\Psi=\left\{\psi^{1}, \psi^{2}, \ldots, \psi^{L}\right\} \subset L^{2}\left(\mathbb{R}^{+}\right)$, define the wavelet system

$$
X(\Psi)=\left\{\psi_{l, j, k}: 1 \leq l \leq L, j \in \mathbb{Z}, k \in \mathbb{Z}^{+}\right\}
$$

where $\psi_{l, j, k}(x)=p^{j / 2} \psi_{l}\left(p^{j} x \ominus k\right)$. The wavelet system $X(\Psi)$ is called a wavelet frame if there exist positive numbers $A$ and $B$ with $0<A \leq B<\infty$ such that

$$
A\|f\|_{2}^{2} \leq \sum_{l=1}^{L} \sum_{j \in \mathbb{Z}} \sum_{k \in \mathbb{Z}^{+}}\left|\left\langle f, \psi_{l, j, k}\right\rangle\right|^{2} \leq B\|f\|_{2}^{2}
$$

for all $f \in L^{2}\left(\mathbb{R}^{+}\right)$. The largest $A$ and the smallest $B$ for which (10) holds are called frame bounds. A frame is a tight frame if $A$ and $B$ are chosen so that $A=B$ and is a normalized tight frame if $A=B=1$.

The collection $X(\Psi)$ is said to be a semi-orthogonal wavelet frame if

$$
\left\langle\psi_{l, j, k}, \psi_{l, j^{\prime}, k^{\prime}}\right\rangle=0 \text { whenever } j \neq j^{\prime} \text { and } k, k^{\prime} \in \mathbb{Z}^{+}, 1 \leq l \leq L
$$

The characterization of wavelet frames on positive half-line has been studied in detail by Abdullah [2].

Theorem 1. Suppose $\Psi=\left\{\psi^{1}, \psi^{2}, \ldots, \psi^{L}\right\} \subset L^{2}\left(\mathbb{R}^{+}\right)$. The affine system $X(\Psi)$ is a tight frame with constant 1 for $L^{2}\left(\mathbb{R}^{+}\right)$, i.e.,

$$
\|f\|_{2}^{2}=\sum_{l=1}^{L} \sum_{j \in \mathbb{Z}} \sum_{k \in \mathbb{Z}^{+}}\left|<f, \psi_{l, j, k}>\right|^{2} \text { for all } f \in L^{2}\left(\mathbb{R}^{+}\right)
$$

if and only if

$$
\sum_{l=1}^{L} \sum_{j \in \mathbb{Z}}\left|\hat{\psi}_{l}\left(p^{j} \xi\right)\right|^{2}=1 \text { for a. e. } \xi \in \mathbb{R}^{+}
$$


and

$$
\sum_{l=1}^{L} \sum_{j=0}^{\infty} \hat{\psi}_{l}\left(p^{j} \xi\right) \overline{\hat{\psi}_{l}\left(p^{j}(\xi \oplus s)\right)}=0 \text { for a. e. } \xi \in \mathbb{R}^{+} \text {and for all } s \in \mathbb{Z}^{+} \backslash p \mathbb{Z}^{+}
$$

In particular, $\Psi$ is a set of basic wavelets of $L^{2}\left(\mathbb{R}^{+}\right)$if and only if $\left\|\psi^{l}\right\|_{2}=1$ for $l=1,2, \ldots, L$ and (11) and (12) hold.

Definition 1. The collection $\left\{\psi_{l}(. \ominus k): 1 \leq l \leq L, k \in \mathbb{Z}^{+}\right\}$forms a wavelet frame for $W_{0}$ if and only if there exist positive numbers $A$ and $B$ such that

$$
A \leq \sum_{k \in \mathbb{Z}^{+}}\left|\hat{\psi}_{l}(\xi \ominus k)\right|^{2} \leq B \text { a.e. } \xi \in S_{l},
$$

where $B=\max B_{l}, A=\min A_{l}$ and $S_{l}=\left\{\xi \in[0,1]: \hat{\psi}_{l}(\xi \ominus k) \neq 0\right\}$.

\section{Semi-orthogonal wavelet frames on positive half-line}

In this section, we characterize semi-orthogonal wavelet frames on positive half-line by virtue of the Walsh-Fourier transform. Our results generalize the characterization of wavelets on Euclidean spaces by means of two basic equations.

For each $l=1,2, \ldots, L$, we define

$$
\hat{\psi}_{l}^{*}(\xi)= \begin{cases}\frac{\hat{\psi}_{l}(\xi)}{\left\|\hat{\psi}_{l}(\xi \ominus k)\right\|_{l^{2}\left(\mathbb{Z}^{+}\right)}} & \text {if } \xi \in S_{l}, \\ 0 & \text { otherwise, }\end{cases}
$$

where $S_{l}=\left\{\xi \in[0,1]: \hat{\psi}_{l}(\xi \ominus k) \neq 0\right\}$. Then, the system $X\left(\Psi^{*}\right)$ obtained by the combined action of dilation and translation of a finite number of functions $\Psi^{*}=\left\{\psi_{1}^{*}, \psi_{2}^{*}, \ldots, \psi_{L}^{*}\right\} \subset L^{2}\left(\mathbb{R}^{+}\right)$is given by

$$
X\left(\Psi^{*}\right)=\left\{\psi_{l, j, k}^{*}: 1 \leq l \leq L, j \in \mathbb{Z}, k \in \mathbb{Z}^{+}\right\}
$$

Theorem 2. Let $X(\Psi)$ and $X\left(\Psi^{*}\right)$ be as defined in (9) and (14), respectively. Then the following statements are equivalent:

(a) $X(\Psi)$ is a semi-orthogonal wavelet frame with frame bounds $A$ and $B$.

(b) For each $l=1,2, \ldots, L$, there exist positive constants $A=\min A_{l}$ and $B=\min B_{l}$ such that inequality (13) holds and

$$
\sum_{l=1}^{L} \sum_{j \in \mathbb{Z}}\left|\hat{\psi}_{l}^{*}\left(p^{j} \xi\right)\right|^{2}=1 \text { for a.e. } \xi \in \mathbb{R}^{+},
$$

and

$$
\sum_{l=1}^{L} \sum_{j=0}^{\infty} \hat{\psi}_{l}^{*}\left(p^{j} \xi\right) \overline{\hat{\psi}_{l}^{*}\left(p^{j}(\xi \oplus s)\right)}=0 \text { for a. e. } \xi \in \mathbb{R}^{+} \text {and for all } s \in \mathbb{Z}^{+} \backslash p \mathbb{Z}^{+} \text {. }
$$

(c) There exist positive numbers $A$ and $B$ such that functions $\psi_{1}, \psi_{2}, \ldots, \psi_{L}$ satisfy conditions (13), (16) and

$$
\begin{gathered}
\sum_{l=1}^{L} \sum_{k \in \mathbb{Z}^{+}} \hat{\psi}_{l}(\xi \oplus k) \overline{\hat{\psi}_{l}\left(p^{j}(\xi \oplus k)\right)}=0 \text { for a.e. } \xi \in \mathbb{R}^{+} j \geq 1, \\
A \leq \sum_{l=1}^{L} \sum_{j \in \mathbb{Z}}\left|\hat{\psi}_{l}\left(p^{j} \xi\right)\right|^{2} \leq B \quad \text { for a.e. } \xi \in \mathbb{R}^{+} .
\end{gathered}
$$


Proof. For each $j \in \mathbb{Z}$, we define

$$
W_{j}=\overline{\operatorname{span}}\left\{\psi_{l, j, k}: 1 \leq l \leq L, k \in \mathbb{Z}^{+}\right\}
$$

and

$$
W_{j}^{*}=\overline{\operatorname{span}}\left\{\psi_{l, j, k}^{*}: 1 \leq l \leq L, k \in \mathbb{Z}^{+}\right\}
$$

(a) $\Rightarrow\left(\right.$ b): Suppose that the affine system $X(\Psi)$ given by (9) is a semi-orthogonal wavelet frame for $L^{2}\left(\mathbb{R}^{+}\right)$with bounds $A$ and $B$, i.e.

$$
A\|f\|^{2} \leq \sum_{l=1}^{L} \sum_{j \in \mathbb{Z}} \sum_{k \in \mathbb{Z}^{+}}\left|\left\langle f, \psi_{l, j, k}\right\rangle\right|^{2} \leq B\|f\|^{2}, \text { for all } f \in L^{2}\left(\mathbb{R}^{+}\right) .
$$

By the scaling property of $W_{j}$ spaces,we have

$$
\sum_{l=1}^{L} \sum_{j \in \mathbb{Z}} \sum_{k \in \mathbb{Z}^{+}}\left|\left\langle f, \psi_{l, j, k}\right\rangle\right|^{2}=\sum_{l=1}^{L} \sum_{k \in \mathbb{Z}^{+}}\left|\left\langle f, \psi_{l, 0, k}\right\rangle\right|^{2}, \quad \text { for all } f \in W_{0} .
$$

Therefore, we have

$$
A\|f\|^{2} \leq \sum_{l=1}^{L} \sum_{k \in \mathbb{Z}^{+}}\left|\left\langle f, \psi_{l, 0, k}\right\rangle\right|^{2} \leq B\|f\|^{2}, \quad \text { for all } f \in W_{0}
$$

which is equivalent to (13).

Since the $W_{j}^{*}$ are orthogonal to each other, i.e., $W_{j_{1}}^{*} \perp W_{j_{2}}^{*}, j_{1} \neq j_{2}$, we have

$$
\sum_{k \in \mathbb{Z}^{+}}\left|\hat{\psi}_{l}^{*}(\xi \ominus k)\right|^{2}=1 \text { for a.e. } \xi \in S_{l} \text {. }
$$

Therefore, the system $X\left(\Psi^{*}\right)$ is a tight frame with frame bound 1 for $L^{2}\left(\mathbb{R}^{+}\right)$. Hence (15) and (16) are satisfied by Theorem 1.

(b) $\Rightarrow$ (c): For each $l=1,2, \ldots, L$, we use equation (13), we have

$$
\frac{1}{B_{l}}\left|\hat{\psi}_{l}(\xi)\right|^{2} \leq\left|\hat{\psi}_{l}^{*}(\xi)\right|^{2} \leq \frac{1}{A_{l}}\left|\hat{\psi}_{l}(\xi)\right|^{2} .
$$

Or equivalently,

$$
\sum_{l=1}^{L} \sum_{j \in \mathbb{Z}} \frac{1}{B_{l}}\left|\hat{\psi}_{l}\left(p^{j} \xi\right)\right|^{2} \leq \sum_{l=1}^{L} \sum_{j \in \mathbb{Z}}\left|\hat{\psi}_{l}^{*}\left(p^{j} \xi\right)\right|^{2} \leq \sum_{l=1}^{L} \sum_{j \in \mathbb{Z}} \frac{1}{A_{l}}\left|\hat{\psi}_{l}\left(p^{j} \xi\right)\right|^{2} .
$$

By taking $\max B_{l}=B, \min A_{l}=A$ and applying (15), we get

$$
A \leq \sum_{l=1}^{L} \sum_{j \in \mathbb{Z}}\left|\hat{\psi}_{l}\left(p^{j} \xi\right)\right|^{2} \leq B \quad \text { a.e. } \xi \in \mathbb{R}^{+}
$$

which shows (18). Moreover, it follows from (15) and (16) that the system $\left\{\psi_{l, 0, k}^{*}: k \in \mathbb{Z}^{+}\right\}$forms a tight frame for $W_{0}^{*}$ with frame bound 1 . By Theorem $1,\left\{\psi_{l, j, k}^{*}: j \in \mathbb{Z}, k \in \mathbb{Z}^{+}\right\}$is a normalized tight frame for $L^{2}\left(\mathbb{R}^{+}\right)$. Since each $\psi_{l}^{*}$ lies in $W_{0}^{*}$, it follows from the tightness of both systems $\left\{\psi_{l, 0, k}^{*}: k \in \mathbb{Z}^{+}\right\}$and $\left\{\psi_{l, j, k}^{*}: j \in \mathbb{Z}, k \in \mathbb{Z}^{+}\right\}$that

$$
\left\|\psi_{l}^{*}\right\|_{2}^{2}=\sum_{j \in \mathbb{Z}} \sum_{k \in \mathbb{Z}^{+}}\left|\left\langle\psi_{l}^{*}, \psi_{l, j, k}^{*}\right\rangle\right|^{2}=\sum_{k \in \mathbb{Z}^{+}}\left|\left\langle\psi_{l}^{*}, \psi_{l, 0, k}^{*}\right\rangle\right|^{2}
$$


Therefore, $\left\langle\psi_{l}^{*}, \psi_{l, j, k}^{*}\right\rangle=0$ for $j \neq 0$. Also, for each $l=1,2, \ldots, L$ and $j \in \mathbb{N}$, we have

$$
\begin{aligned}
0 & =\left\langle\psi_{l}^{*}, \psi_{l, j, k}^{*}\right\rangle=\left\langle\hat{\psi}_{l}^{*}, \hat{\psi}_{l, j, k}^{*}\right\rangle \\
& =p^{j / 2} \int_{\mathbb{R}^{+}} \hat{\psi}_{l}^{*}(\xi) \overline{\hat{\psi}_{l}^{*}\left(p^{-j \xi) \chi\left(k, p^{-j} \xi\right)} d \xi\right.} \\
& =p^{-j / 2} \int_{\mathbb{R}^{+}} \hat{\psi}_{l}^{*}\left(p^{j} \xi\right) \overline{\hat{\psi}_{l}^{*}(\xi) \chi(k, \xi)} d \xi \\
& =p^{-j / 2} \sum_{r \in \mathbb{Z}^{+}} \int_{r[0,1]} \hat{\psi}_{l}^{*}\left(p^{j \xi}\right) \overline{\hat{\psi}_{l}^{*}(\xi) \chi(k, \xi)} d \xi \\
& =p^{-j / 2} \int_{[0,1]}\left\{\sum_{r \in \mathbb{Z}^{+}} \hat{\psi}_{l}^{*}\left(p^{j}(\xi \oplus r)\right) \overline{\hat{\psi}_{l}^{*}(\xi \oplus r)}\right\} \overline{\chi(k, \xi)} d \xi .
\end{aligned}
$$

This shows that

$$
\sum_{r \in \mathbb{Z}^{+}} \hat{\psi}_{l}^{*}(\xi \oplus r) \overline{\hat{\psi}_{l}^{*}\left(p^{j}(\xi \oplus r)\right)}=0 \text { for a.e. } \xi \in \mathbb{R}^{+}, j \geq 1
$$

Therefore, we have

$$
\sum_{k \in \mathbb{Z}^{+}} \hat{\psi}_{l}(\xi \oplus k) \overline{\hat{\psi}_{l}\left(p^{j}(\xi \oplus k)\right)}=\left\{\sum_{k \in \mathbb{Z}^{+}}\left|\hat{\psi}_{l}\left(p^{j}(\xi \ominus k)\right)\right|^{2}\right\}^{\frac{1}{2}}\left\{\sum_{k \in \mathbb{Z}^{+}}\left|\hat{\psi}_{l}(\xi \ominus k)\right|^{2}\right\}^{\frac{1}{2}} \times \sum_{k \in \mathbb{Z}^{+}} \hat{\psi}_{l}^{*}(\xi \oplus k) \overline{\hat{\psi}_{l}^{*}\left(p^{j}(\xi \oplus k)\right)}=0 .
$$

Hence, condition (17) is satisfied.

(c) $\Rightarrow\left(\right.$ a): we use condition (13)which shows that the affine system $X(\Psi)$ constitutes a frame for $W_{j}$. Moreover, identity (17) shows that $W_{0}$ is orthogonal to $W_{j}$ for $j \neq 0$. Therefore, by means of change of variables, we have

$$
\left\langle\psi_{l, j, k}, \psi_{l, n, m}\right\rangle=\left\langle\psi_{l, 0, k-p^{1-j} m}, \psi_{l, n-j, 0}\right\rangle, \quad 1 \leq l \leq L, k, m \in \mathbb{Z}^{+}
$$

It is immediate from the above relation that $W_{j} \perp W_{n}$ for $j \neq n$. Thus, we conclude that $X(\Psi)$ forms a frame for $W=$ $\overline{\operatorname{span}}\left\{\psi_{l, j, k}: 1 \leq l \leq L, j \in \mathbb{Z}, k \in \mathbb{Z}^{+}\right\}$. Next, we claim that $W=L^{2}\left(\mathbb{R}^{+}\right)$. It suffices to show that the system $X\left(\Psi^{*}\right)$ given by (14) is a frame for $L^{2}\left(\mathbb{R}^{+}\right)$. We set

$$
S^{0}=\left\{f \in S: \sup \hat{f} \subset \mathbb{R}^{+}\{0\}\right\}
$$

which is dense in $L^{2}\left(\mathbb{R}^{+}\right)$. Let $f$ be in $S^{0}$. Applying Parseval's formula, we obtain

$$
\begin{aligned}
\sum_{j \in \mathbb{Z}} \sum_{k \in \mathbb{Z}^{+}}\left|\left\langle f, \psi_{l, j, k}^{*}\right\rangle\right|^{2}=\sum_{j \in \mathbb{Z}} \sum_{k \in \mathbb{Z}^{+}}\left|\left\langle\hat{f}, \hat{\psi}_{l, j, k}^{*}\right\rangle\right|^{2} & =\sum_{j \in \mathbb{Z}} \sum_{k \in \mathbb{Z}^{+}} p^{j / 2}\left|\int_{\mathbb{R}^{+}} \hat{f}(\xi) \overline{\hat{\psi}_{l}^{*}\left(p^{-j} \xi\right) \overline{\chi\left(k, p^{-j} \xi\right.}}\right|^{2} d \xi \\
& =\sum_{j \in \mathbb{Z}} p^{-j / 2} \sum_{k \in \mathbb{Z}^{+}}\left|\int_{\mathbb{R}^{+}} \hat{f}\left(p^{j} \xi\right) \overline{\hat{\psi}_{l}^{*}(\xi)} \chi(k, \xi)\right|^{2} d \xi \\
& =\sum_{j \in \mathbb{Z}} p^{-j / 2} \int_{[0,1]}\left|\sum_{s \in \mathbb{Z}^{+}} \hat{f}\left(p^{j}(\xi \oplus s)\right) \overline{\hat{\psi}_{l}^{*}(\xi \oplus s)}\right|^{2} d \xi \\
& =\sum_{j \in \mathbb{Z}} p^{-j / 2} \int_{[0,1]}\left|F_{j}^{l}(\xi)\right|^{2} d \xi
\end{aligned}
$$

where

$$
F_{j}^{l}(\xi)=\sum_{s \in \mathbb{Z}^{+}} \hat{f}\left(p^{j}(\xi \oplus s)\right) \overline{\hat{\psi}_{l}^{*}(\xi \oplus s)}, l=1,2, \ldots, L
$$


Moreover, we have

$$
\begin{aligned}
\int_{[0,1]}\left|F_{j}^{l}(\xi)\right|^{2} d \xi & =\int_{[0,1]}\left|\sum_{s \in \mathbb{Z}^{+}} \hat{f}\left(p^{j}(\xi \oplus s)\right) \overline{\hat{\psi}_{l}^{*}(\xi \oplus s)}\right|^{2} d \xi \\
& \leq \int_{[0,1]} \sum_{s \in \mathbb{Z}^{+}}\left|\hat{f}\left(p^{j}(\xi \oplus s)\right) \overline{\hat{\psi}_{l}^{*}(\xi \oplus s)}\right|^{2} d \xi \\
& =\sum_{s \in \mathbb{Z}^{+}} \int_{[0,1]}\left|\hat{f}\left(p^{j}(\xi \oplus s)\right) \overline{\hat{\psi}_{l}^{*}(\xi \oplus s)}\right|^{2} d \xi \\
& =\int_{\mathbb{R}^{+}}\left|\hat{f}\left(p^{j} \xi\right) \overline{\hat{\psi}_{l}^{*}(\xi)}\right|^{2} d \xi \\
& \leq\left(\int_{\mathbb{R}^{+}}\left|\hat{f}\left(p^{j} \xi\right)\right|^{2} d \xi\right)^{\frac{1}{2}}\left(\int_{\mathbb{R}^{+}}\left|\hat{\psi}_{l}^{*}(\xi)\right|^{2} d \xi\right)^{\frac{1}{2}} .
\end{aligned}
$$

Since both $f$ and $\hat{f}$ are compactly supported and each $\psi_{l}^{*}$ lies in $L^{2}\left(\mathbb{R}^{+}\right)$, it follows from the above inequality that $F_{j}^{l}(\xi) \in L^{2}([0,1])$. Also, for any $j \in \mathbb{Z}$, we have

$$
\left|F_{j}^{l}(\xi)\right|^{2} \leq \sum_{s \in \mathbb{Z}^{+}}\left|\hat{f}\left(p^{j}(\xi \oplus s)\right)\right|^{2} \sum_{s \in \mathbb{Z}^{+}}\left|\hat{\psi}_{l}^{*}(\xi \oplus s)\right|^{2}, \quad l=1,2, \ldots, L
$$

and

$$
\int_{\mathbb{R}^{+}} \hat{f}\left(p^{j} \xi\right) \overline{\hat{\psi}_{l}^{*}(\xi)} \chi(k, \xi) d \xi=\int_{[0,1]} F_{j}^{l}(\xi) \chi(k, \xi) d \xi .
$$

By applying the Plancherel formula, we get

$$
\sum_{k \in \mathbb{Z}^{+}}\left|\int_{[0,1]} F_{j}^{l}(\xi) \chi(k, \xi) d \xi\right|^{2}=\int_{[0,1]}\left|F_{j}^{l}(\xi)\right|^{2} d \xi .
$$

Hence equation(20) becomes

$$
\begin{aligned}
\sum_{j \in \mathbb{Z}} \sum_{k \in \mathbb{Z}^{+}}\left|\left\langle f, \boldsymbol{\psi}_{l, j, k}^{*}\right\rangle\right|^{2} & =\sum_{j \in \mathbb{Z}} p^{-j / 2} \int_{[0,1]}\left|\sum_{s \in \mathbb{Z}^{+}} \hat{f}\left(p^{j}(\xi \oplus s)\right) \overline{\hat{\psi}_{l}^{*}(\xi \oplus s)}\right|^{2} d \xi \\
& =\sum_{j \in \mathbb{Z}} p^{-j / 2} \int_{[0,1]} \sum_{s \in \mathbb{Z}^{+}} \overline{\hat{f}\left(p^{j}(\xi \oplus s)\right)} \hat{\psi}_{l}^{*}(\xi \oplus s) \sum_{s \in \mathbb{Z}^{+}} \hat{f}\left(p^{j}(\xi \oplus s)\right) \overline{\hat{\psi}_{l}^{*}(\xi \oplus s)} d \xi \\
& =\sum_{j \in \mathbb{Z}} p^{-j / 2} \int_{\mathbb{R}^{+}} \overline{\hat{f}\left(p^{j} \xi\right)} \hat{\psi}_{l}^{*}(\xi) \sum_{s \in \mathbb{Z}^{+}} \hat{f}\left(p^{j}(\xi \oplus s)\right) \overline{\hat{\psi}_{l}^{*}(\xi \oplus s)} d \xi \\
& =\int_{\mathbb{R}^{+}}|\hat{f}(\xi)|^{2} \sum_{j \in \mathbb{Z}}\left|\hat{\psi}_{l}^{*}\left(p^{-j} \xi\right)\right|^{2} d \xi+R(f),
\end{aligned}
$$

where

$$
R(f)=\sum_{j \in \mathbb{Z}} \int_{\mathbb{R}^{+}} \overline{\hat{f}(\xi)} \hat{\psi}_{l}^{*}\left(p^{-j} \xi\right) \sum_{s \in \mathbb{Z}^{+}} \hat{f}\left(\xi \oplus p^{-j} s\right) \overline{\hat{\psi}_{l}^{*}\left(p^{-j} \xi \oplus s\right)} d \xi .
$$

For given $s \in \mathbb{Z}^{+}$, there is a unique pair $(k, m)$ with $k \in \mathbb{Z}^{+}$and $m \in \mathbb{Z}^{+} \backslash p \mathbb{Z}^{+}$, such that $s=p^{k} m$. Since $R(f)$ is absolutely convergent, we can estimate $R(f)$ by rearranging the series, changing the order of summation and integration by the Levi 
lemma as follows

$$
\begin{aligned}
& R(f)=\sum_{j \in \mathbb{Z}} \int_{\mathbb{R}^{+}} \overline{\hat{f}(\xi)} \hat{\psi}_{l}^{*}\left(p^{-j} \xi\right)\left\{\sum_{s \in \mathbb{Z}^{+}} \hat{f}\left(\xi \oplus p^{-j} s\right) \overline{\hat{\psi}_{l}^{*}\left(p^{-j \xi} \oplus s\right)}\right\} d \xi \\
& =\sum_{j \in \mathbb{Z}} \int_{\mathbb{R}^{+}} \overline{\hat{f}(\xi)}\left\{\sum_{k \in \mathbb{Z}^{+}} \sum_{m \in \mathbb{Z}^{+} \backslash p \mathbb{Z}^{+}} \hat{\psi}_{l}^{*}\left(p^{-j} \xi\right) \hat{f}\left(\xi \oplus p^{-j} p^{k} m\right) \overline{\hat{\psi}_{l}^{*}\left(p^{-j} \xi \oplus p^{k} m\right)}\right\} d \xi \\
& =\int_{\mathbb{R}^{+}} \overline{\hat{f}(\xi)}\left\{\sum_{k \in \mathbb{Z}^{+}} \sum_{m \in \mathbb{Z}^{+} \backslash p \mathbb{Z}^{+}} \sum_{j \in \mathbb{Z}} \hat{\psi}_{l}^{*}\left(p^{-j+k} \xi\right) \hat{f}\left(\xi \oplus p^{j} m\right) \overline{\hat{\psi}_{l}^{*}\left(p^{-j+k} \xi \oplus p^{k} m\right)}\right\} d \xi \\
& =\int_{\mathbb{R}^{+}} \overline{\hat{f}(\xi)}\left\{\sum_{j \in \mathbb{Z}} \sum_{m \in \mathbb{Z}^{+} \backslash p \mathbb{Z}^{+}} \hat{f}\left(\xi \oplus p^{j} m\right) \sum_{k \in \mathbb{Z}^{+}} \hat{\psi}_{l}^{*}\left(p^{-j+k} \xi \overline{\hat{\psi}_{l}^{*}\left(p^{k}\left(p^{-j} \xi \oplus m\right)\right)}\right\} d \xi\right. \\
& =\sum_{j \in \mathbb{Z}} \sum_{m \in \mathbb{Z}^{+} \backslash p \mathbb{Z}^{+}} \int_{\mathbb{R}^{+}} \overline{\hat{f}(\xi)} \hat{f}\left(\xi \oplus p^{j} m\right) \sum_{k \in \mathbb{Z}^{+}} \hat{\psi}_{l}^{*}\left(p^{-j+k} \xi\right) \overline{\hat{\psi}_{l}^{*}\left(p^{k}\left(p^{-j} \xi \oplus m\right)\right)} d \xi .
\end{aligned}
$$

Using the above estimate of $R(f)$ in $(21)$, we obtain

$$
\begin{aligned}
\sum_{l=1}^{L} \sum_{j \in \mathbb{Z}} \sum_{k \in \mathbb{Z}^{+}}\left|\left\langle f, \psi_{l, j, k}^{*}\right\rangle\right|^{2} & =\int_{\mathbb{R}^{+}}|\hat{f}(\xi)|^{2} \sum_{l=1}^{L} \sum_{j \in \mathbb{Z}}\left|\hat{\psi}_{l}^{*}\left(p^{-j} \xi\right)\right|^{2} d \xi \\
& +\sum_{j \in \mathbb{Z}} \sum_{m \in \mathbb{Z}^{+} \backslash p \mathbb{Z}^{+}} \int_{\mathbb{R}^{+}} \overline{\hat{f}(\xi)} \hat{f}\left(\xi \oplus p^{j} m\right) \sum_{l=1}^{L} \sum_{k \in \mathbb{Z}^{+}} \hat{\psi}_{l}^{*}\left(p^{-j+k} \xi\right) \overline{\hat{\psi}_{l}^{*}\left(p^{k}\left(p^{-j} \xi \oplus m\right)\right)} d \xi
\end{aligned}
$$

From (16), (19) and (22), we have

$$
A / B\|f\|_{2}^{2} \leq \sum_{l=1}^{L} \sum_{j \in \mathbb{Z}} \sum_{k \in \mathbb{Z}^{+}}\left|\left\langle f, \psi_{l, j, k}^{*}\right\rangle\right|^{2} \leq B / A\|f\|_{2}^{2} .
$$

This means that the system $\left\{\psi_{l, j, k}^{*}: 1 \leq l \leq L, j \in \mathbb{Z}, k \in \mathbb{Z}^{+}\right\}$is a frame for $L^{2}\left(\mathbb{R}^{+}\right)$. Hence, we get the desired result.

\section{Competing interests}

The authors declare that they have no competing interests.

\section{Authors' contributions}

All authors have contributed to all parts of the article. All authors read and approved the final manuscript.

\section{References}

[1] Abdullah, On the characterization of non-uniform wavelet sets on positive half line, J. Info. Comp. Sc., 10 (1) (2015), $046-053$.

[2] Abdullah, Characterization of p-wavelets on positive half line using the Walsh-Fourier transform, Int. J. Anal. Appl., 10 (2) (2016), 77-84.

[3] M. Bownik, On characterizations of multiwavelets in $L^{2}\left(\mathbb{R}^{n}\right)$, Proc. Amer. Math. Soc., 129 (2001), 3265-3274.

[4] A. Calogero, A characterization of wavelets on general lattices, J. Geom. Anal., 10 (2000), 597-622.

[5] O. Christensen, An Introduction to Frames and Riesz Bases, Birkhäuser, Boston, 2015.

[6] I. Daubechies, A. Grossmann, Y. Meyer, Painless non-orthogonal expansions, J. Math. Phys., 27 (5) (1986), 1271-1283. 
[7] R.J. Duffin, A.C. Shaeffer, A class of nonharmonic Fourier series, Trans. Am. Math. Soc., 72 (1952), 341-366.

[8] Y. A. Farkov, Orthogonal p-wavelets on R+, in Proceedings of International Conference Wavelets and Splines, St. Petersberg State University, St. Petersberg, (2005), 4-26.

[9] Y. A. Farkov, A. Y. Maksimov and S. A. Stroganov, On biorthogonal wavelets related to the Walsh functions, Int. J. Wavelets, Multiresolut. Inf. Process., 9(3) (2011), 485-499.

[10] S. Goyal and F. A. Shah, Minimum-energy wavelet frames generated by the Walsh polynomials, Cogent Mathematics (2015), 2 : 1114830, http://dx.doi.org/10.1080/23311835.2015.1114830.

[11] G. Gripenberg, A necessary and sufficient condition for the existence of a father wavelet, Stud. Math., 114 (1995), $207-226$.

[12] E. Hernández and G. Weiss, A First Course on Wavelets, CRC Press, New York, 1996.

[13] H. O. Kim, R. Y. Kim and J. K. Lim, Semi-orthogonal frame wavelets and frame multiresolution analysis, Bull. Australian Math. Soc., 65 (2002), 35-44.

[14] A. Ron and Z. Shen, Frames and stable bases for shift invariant subspaces of $L^{2}\left(\mathbb{R}^{d}\right)$, Canad. J. Math., 47 (1995), $1051-1094$.

[15] F A Shah and L. Debnath, Dyadic wavelet frames on a half-line using the Walsh-Fourier transform, Integ. Trans. Special Funct., 22(7) (2011), 477-486.

[16] F A Shah and L. Debnath, $p$-Wavelet frame packets on a half-line using the Walsh Fourier transform, Integr. Transf. Spec. Funct., 22(12) (2011), 907-912.

[17] F. A. Shah, Tight wavelet frames generated by the Walsh polynomials, Int. J. Wavelets, Multiresolut. Inf. Process., 11(6) (2013) 1350042, (15 pages). 